\title{
Assembly of Helicobacter pylori Initiation Complex Is Determined by Sequence-Specific and Topology-Sensitive DnaA-oriC Interactions
}

\author{
Rafał Donczew ${ }^{1}$, Thorsten Mielke ${ }^{2}$, Paweł Jaworski ${ }^{1}$, \\ Jolanta Zakrzewska-Czerwińska ${ }^{1,3}$ and Anna Zawilak-Pawlik ${ }^{1}$ \\ 1 - Institute of Immunology and Experimental Therapy, Polish Academy of Sciences, Department of Microbiology, Weigla 12, \\ 53-114 Wroclaw, Poland \\ 2 - Max Planck Institute for Molecular Genetics, Ihnestrasse 63-73, 14195 Berlin, Germany \\ 3 - University of Wrocław, Faculty of Biotechnology, Joliot-Curie 14a, 50-138 Wrocław, Poland
}

Correspondence to Rafał Donczew and Anna Zawilak-Pawlik: rafal.donczew@iitd.pan.wroc.pl; zawilak@iitd.pan.wroc.pl http://dx.doi.org/10.1016/j.jmb.2014.05.018

Edited by J. Berger

\begin{abstract}
In bacteria, chromosome replication is initiated by binding of the DnaA initiator protein to DnaA boxes located in the origin of chromosomal replication (oriC). This leads to DNA helix opening within the DNA-unwinding element. Helicobacter pylori oriC, the first bipartite origin identified in Gram-negative bacteria, contains two subregions, oriC1 and oriC2, flanking the $d n a A$ gene. The DNA-unwinding element region is localized in the oriC2 subregion downstream of $d n a A$. Surprisingly, oriC2-DnaA interactions were shown to depend on DNA topology, which is unusual in bacteria but is similar to initiator-origin interactions observed in higher organisms. In this work, we identified three DnaA boxes in the oriC2 subregion, two of which were bound only as supercoiled DNA. We found that all three DnaA boxes play important roles in orisome assembly and subsequent DNA unwinding, but different functions can be assigned to individual boxes. This suggests that the $H$. pylori oriC may be functionally divided, similar to what was described recently for Escherichia coli oriC. On the basis of these results, we propose a model of initiation complex formation in H. pylori.
\end{abstract}

(c) 2014 Elsevier Ltd. All rights reserved.

\section{Introduction}

Replication of genetic material is a fundamental process in all dividing cells. The main regulatory factors act at the initiation step controlling initiation complex formation to guarantee that DNA synthesis commences at the precisely determined moment and that DNA is replicated only once per cell cycle $[1,2]$. In bacteria, the basic initiation mechanism consists of DnaA initiator protein interaction with a specific locus termed oriC to form a nucleoprotein complex, resulting in DNA unwinding within a helically unstable, usually AT-rich, DNA-unwinding element (DUE) region. Bacterial origins are composed of a single DUE and a variable number of DnaA boxes (i.e., sequences bound by DnaA), which are usually grouped into a single cluster. However, there are exceptions in which DnaA boxes are organized into two (e.g., Helicobacter pylori) or three (e.g., Bacillus subtilis) clusters [3,4]. The sequences and distribution of DnaA boxes vary significantly among species and are of utmost importance for the formation of a usually multiprotein complex, which is capable of unwinding DNA (orisome) $[5,6]$.

The most comprehensive understanding of a bacterial replication-initiation mechanism comes from studies on Escherichia coli [7-9]. The E. coli oriC spans about 250 bp between gidA and mioC and contains one cluster of DnaA boxes, which is located downstream of the DUE (Fig. 1a). Multiple DnaA boxes are bound with different affinity and/or specificity by the DnaA protein regardless of the DNA topology [10]. Boxes R1, R2 and R4 (Fig. 1a) are 9-mer double-stranded DnaA boxes with the consensus sequence $5^{\prime}-$ TTATNCACA- $3^{\prime}$. These are usually referred as "strong" sites because of their high affinity for DnaA $\left(K_{d} \approx 5-50 \mathrm{nM}\right)[11,12]$. Strong boxes are bound by both ATP-DnaA and ADP-DnaA 
(a)

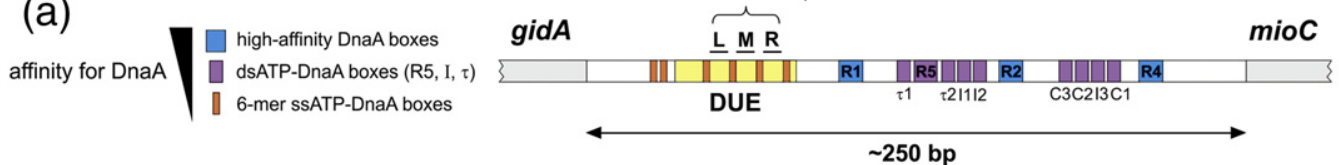

(b)

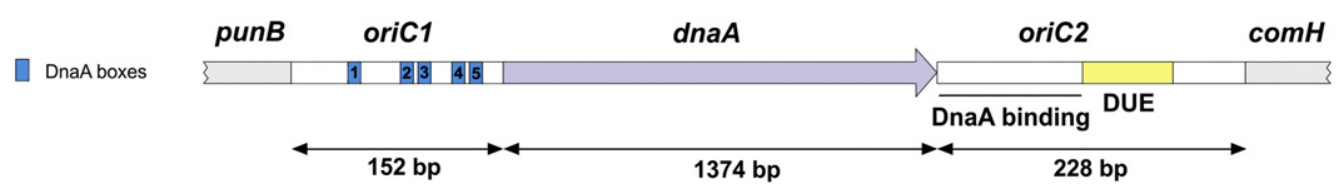

Fig. 1. (a) The structure of the E. coli origin of replication (based mostly on Leonard and Grimwade [9]). DnaA boxes bound with different affinities and specificities are marked. (b) The structure of the $H$. pylori origin of replication based on the last published work (Donczew et al. [4]). DnaA boxes in oriC1 are marked. Identification of oriC2 DnaA boxes was one of the aims of the present work.

with similar affinities, although some studies suggest that this is not the case for box R2 [13-15]. The low-affinity sites in the $E$. coli oriC interact preferentially with ATP-DnaA and are organized into two arrays located between boxes R1-R2 and R2-R4 (Fig. 1a). The three I sites are 9-mer sequences that differ by 3-4 bases from the consensus of highaffinity boxes [16]. The two 9-mer t sites are even less similar to R-boxes, but they share a 6-mer consensus TGATCC sequence with 12 and 13 sites [14]. Additional low-affinity sites include 9-mer boxes R5, C1, C2 and C3. Boxes C2 and C3 overlap box $\mathrm{R} 3$, which is probably not involved in DnaA protein binding $[9,17]$. In addition to the low-affinity doublestranded ATP-DnaA boxes, there are six 6-mer, single-stranded ATP-DnaA boxes with a consensus AGATCT sequence. These boxes are located in the DUE or at its border and are bound only as single-stranded DNA (ssDNA) (Fig. 1a) [18]. Furthermore, the DUE region contains two other sequences important for SsDNA-DnaA interactions: TTGT in 13-mer $M$ and TTATT in 13-mer $R$ [15] (Fig. 1a). During the initiation event, ATP-DnaA protein, assisted by the DiaA protein, is recruited to low-affinity sites at oriC through cooperative interactions with DnaA molecules situated at high-affinity sites $[19,20]$. It has been suggested that boxes R1, $\mathrm{R} 2$ and R4, which are occupied by DnaA protein throughout the cell cycle [21], form a sort of molecular anchor for DnaA molecules that gradually bind to low-affinity sites $[20,22]$. Finally, with the help of the IHF protein, DnaA molecules oligomerize and it was proposed that they assemble into a righthanded helical filament $[23,24]$. According to the model, the DNA strand is wrapped around the protein filament, introducing strong torsional stress that leads to unwinding of the DUE [25]. The DnaA oligomer is apparently divided into two functional parts that differ with respect to the activity of their DnaA molecules [26]. DnaA molecules bound at the left half of oriC (near the DUE) are involved in DUE unwinding and helicase loading, whereas molecules bound at the right-half of oriC facilitate helicase binding and loading $[26,27]$. Additionally, the right half of oriC was shown to be redundant for slow-growing cells but necessary for rapid growth in rich media [28]. The main pathways controlling initiation of $E$. coli chromosome replication involve regulation of ATP-DnaA level by the RIDA (regulatory inactivation of $D n a A$ )/DARS ( $D$ naA-reactivating sequence) system, control of free cytoplasmic DnaA protein level and introduction of steric obstacles by SeqA and Fis in the oriC region, which hinders DnaA-oriC interactions [29-31].

The $H$. pylori oriC region is bipartite in structure and is the first such origin identified in Gramnegative bacteria [4]. It is composed of oriC1 and oriC2 subregions separated by the $d n a A$ gene and the DUE region, which is located in oriC2 between 120 and $170 \mathrm{bp}$ downstream of the $d n a A$ gene (Fig. 1b). The DnaA protein binds to both oriC1 and oriC2, bridging them together and looping out $d n a A$ [4]. There are five DnaA boxes located at oriC1. The affinity of DnaA protein is highest for the pairs of boxes c2-c3 and c4-c5, whereas the affinity toward box $\mathrm{c} 1$ is the lowest of all the boxes [32,33]. The consensus sequence of oriC1 boxes is $T(C / T)$ ATTCACN with the exception of box $\mathrm{c} 1$, which differs from this consensus by two bases. DnaA binding to oriC1 is significantly enhanced in the presence of HobA, which interacts with the N-terminus of DnaA and plays a role in orisome formation similar to that of DiaA in E. coli $[34,35]$. Electron microscopy (EM) analyses have revealed that DnaA is bound to the oriC2 region between the $d n a A$ gene and the DUE. In contrast to oriC1, which is bound by DnaA regardless of DNA topology, DnaA protein interacts with oriC2 only when this subregion is supercoiled. Furthermore, DnaA preferentially interacts with supercoiled oriC2 and apparently subsequently adjoins oriC1 to the DnaA-oriC2 complex [4]. It has also been found that oriC2 is susceptible to DnaA-dependent DNA unwinding in the absence of oriC1, but the size of the initiation bubble and the 
level of unwinding are much lower compared with those of the bipartite oriC region. Taken together, these findings suggest that formation of the nucleoprotein initiation complex on the bipartite $H$. pylori oriC is ordered and highly structured, most likely by the layout and affinity of the DnaA boxes at oriC1 and oriC2 subregions.

The aim of this study was to identify and characterize the DnaA boxes involved in DnaAoriC2 interactions, with a specific focus on topology-dependent binding. By applying dimethyl sulfate (DMS) footprinting, we discovered three novel DnaA boxes in oriC2, two of which are bound only when oriC2 is negatively supercoiled. Additionally, we discovered a short region close to the DUE that is hypersensitive to DMS modification, which might be an indication of alterations in the DNA structure upon orisome formation. The roles of individual identified sequences in orisome formation and DNA unwinding were confirmed experimentally by an analysis of DnaA binding to oriC2 containing mutated DnaA boxes. On the basis of the results of this and previous studies, we propose a model of orisome assembly in $H$. pylori.

\section{Results}

\section{Identification of DnaA boxes in the oriC2 subregion and determination of topology-sensitive DnaA-oriC interactions}

In previous work, we showed that oriC2 specifically unwinds at the DUE upon interaction with DnaA protein, both in vitro and in vivo. However, DnaA was found to interact with oriC2 only when the region was negatively supercoiled, leading to the conclusion that DnaA protein binding to oriC2 is dependent on DNA topology. In order to identify DnaA binding sites at the supercoiled $H$. pylori oriC region and to assess the influence of DNA superhelicity on DnaA interactions with particular DnaA boxes in each of the oriC1 and oriC2 subregions, we applied DMS footprinting. This method allows us to detect protein-DNA interactions and identify protein binding sites without damaging sugar-phosphate backbone of DNA, which thus retains its native topology (see also Discussion). DMS modifies $G$ residues, making the proximate phosphodiester bond of the DNA backbone susceptible to piperidine cleavage as in Maxam-Gilbert sequencing; proteins bound to DNA protect nucleic acids in the binding region from DMS modification. A subsequent primer extension (PE) analysis of the piperidinecleaved DNA allows identification of protein binding sites.

Supercoiled pori1ori2 plasmid (Table S1) was incubated with increasing concentrations of DnaA protein and treated with DMS; a reaction containing no DnaA served as a control. To precisely map the oriC2 subregion, we identified binding sites by $P E$ reactions using two primers complementary to the lower strand (according to the published $H$. pylori 26695 genome sequence [36]) (P-2 and P-3; Table S2 and Fig. 3a). We detected DnaA-dependent protection from DMS modification of six $G$ residues in oriC2 (Figs. 2 and 3a). A comparison of the DNA sequence in the vicinity of the protected nucleotides with the known sequences of $H$. pylori DnaA boxes at oriC1 revealed three novel DnaA binding sites in the oriC2 region: box c6 (TCATTCACT), ts 1 (TCATTCCAT) and ts2 (CCATTCACG) (Figs. 2 and 3a). The first box is designated "c6", for classic, since it is consistent with the consensus sequence of DnaA boxes at oriC1 and is recognized as both linear and supercoiled forms (consequently, oriC1 boxes are designated c1-c5) [32]; the second two boxes at oriC2 are designated "ts" for topologically sensitive (see also below). Box c6 is located $23 \mathrm{bp}$ from the $3^{\prime}$ end of the $d n a A$ gene, whereas boxes ts are located 57 bp downstream of box c6 and 10 bp upstream of the DUE (Figs. 2 and 3a). These latter boxes constitute a pair of head-to-tail boxes, which are atypically not separated by any base pairs. We also detected a region that becomes hypersensitive to DMS modification upon DnaA binding to oriC2. The hs (hypersensitive) region is located between boxes ts and the DUE. We speculate that this region reflects alterations in the DNA structure introduced by orisome complex assembly, which precedes, and is possibly required, for DUE unwinding.

We next analyzed the differences in DnaA binding to linear and supercoiled subregions by DMS footprinting using the pori1ori2 plasmid. The supercoiled plasmid was linearized by digestion with Scal, which cuts pori1ori2 approximately 700 bp downstream from oriC2 and 1500 bp upstream from oriC1 [4]. Both forms of the plasmid were treated with DMS in the presence or absence of the DnaA protein. The boxes protected by DnaA were subsequently determined by $\mathrm{PE}$ using primers complementary to the lower strand (P-1, P-2 and P-3; Table $\mathrm{S} 2$ and Fig. 3a). In the case of oriC1, we confirmed the positions of four of the five DnaA boxes identified in a previous study (Fig. 3a and Fig. S1) [32]. We did not observe any protection from DMS modification at the weak box c1. As suggested by the results of previous experiments, we found that DnaA interactions with all of the oriC1 boxes were independent of DNA topology (Fig. 4 and Fig. S2). For oriC2, we detected binding at box $\mathrm{c} 6$ in both linear and supercoiled forms (Fig. 4 and Fig. S2). The interaction with box c6 is probably weak since no interaction of DnaA with linear oriC2 was found in previous gel shift, surface plasmon resonance or EM experiments [4]. In marked contrast, the interaction of DnaA with the pair of boxes ts located next to the DUE was entirely dependent on DNA topology: only the supercoiled and 


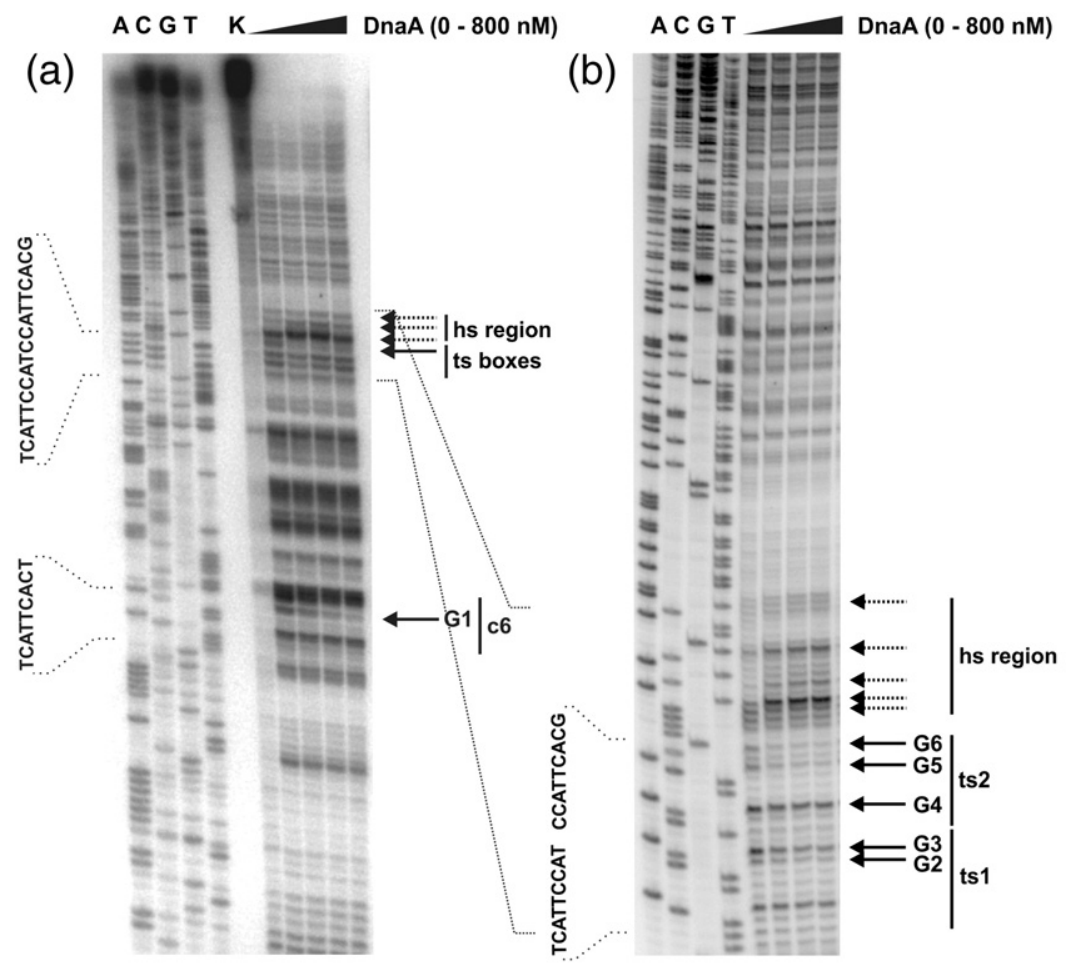

(c)

box c6:

G:

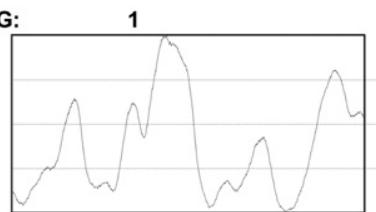

0 nM DnaA
$200 \mathrm{nM}$ DnaA

1

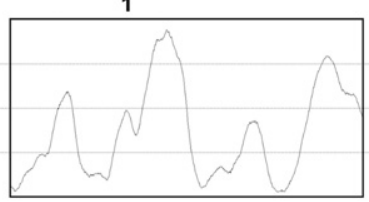

$400 \mathrm{nM}$ DnaA

1

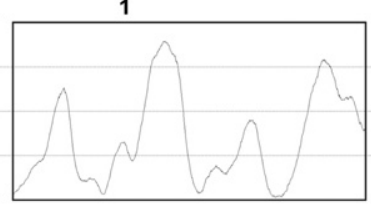

$800 \mathrm{nM}$ DnaA

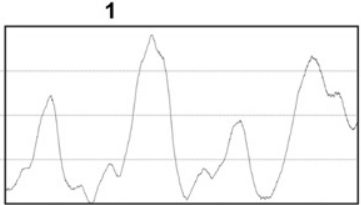

boxes ts:

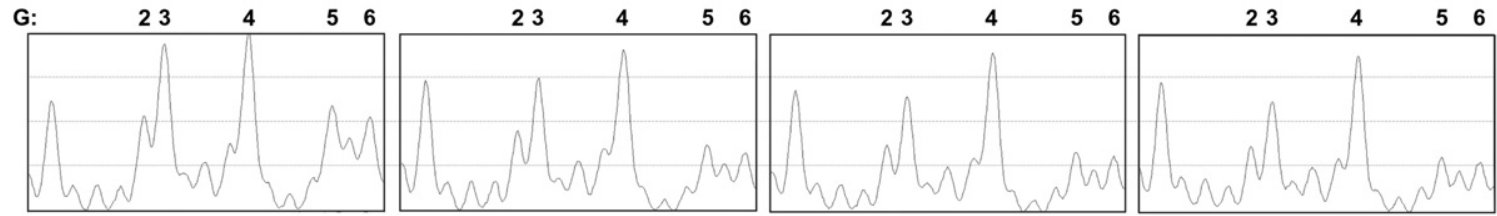

Fig. 2. Identification of DnaA boxes in the oriC2 subregion of the $H$. pylori origin of replication. Plasmid pori1ori2 was incubated with the indicated amounts of DnaA protein $(0,200,400$ and $800 \mathrm{nM})$, treated with DMS and then used as a substrate for PE analysis. ${ }^{32}$ P-labeled primers P-2 (a) and P-3 (b) are complementary to the lower strand. The first lane in the left-hand picture (a) was obtained for a plasmid incubated with the protein but not treated with DMS. Continuous-line arrows indicate the nucleotides protected from DMS modification upon protein binding, and dotted-line arrows correspond to the nucleotides that became hypersensitive to DMS upon protein binding. The positions of the identified boxes are marked next to the arrows. The inferred sequences of boxes are presented next to the sequencing lanes. Please refer to Fig. $3 a$ for details regarding the structure and sequence of the $H$. pylori oriC region. (c) Densitometric plots obtained for the lanes corresponding to the indicated amounts of DnaA protein. Protected guanosine residues $(\mathrm{G})$ are marked above the first plot.

not the linear form of these boxes was bound by DnaA. Boxes ts are not equally protected by DnaA from DMS modification. Box ts2 is protected stronger than ts 1 (Fig. 2), which may suggest that DnaA exhibits lower affinity toward box ts 1 than toward box ts2. In addition, the binding of DnaA to linear DNA did not induce the structural change in DNA that resulted in hypersensitivity of this region to DMS modification observed 


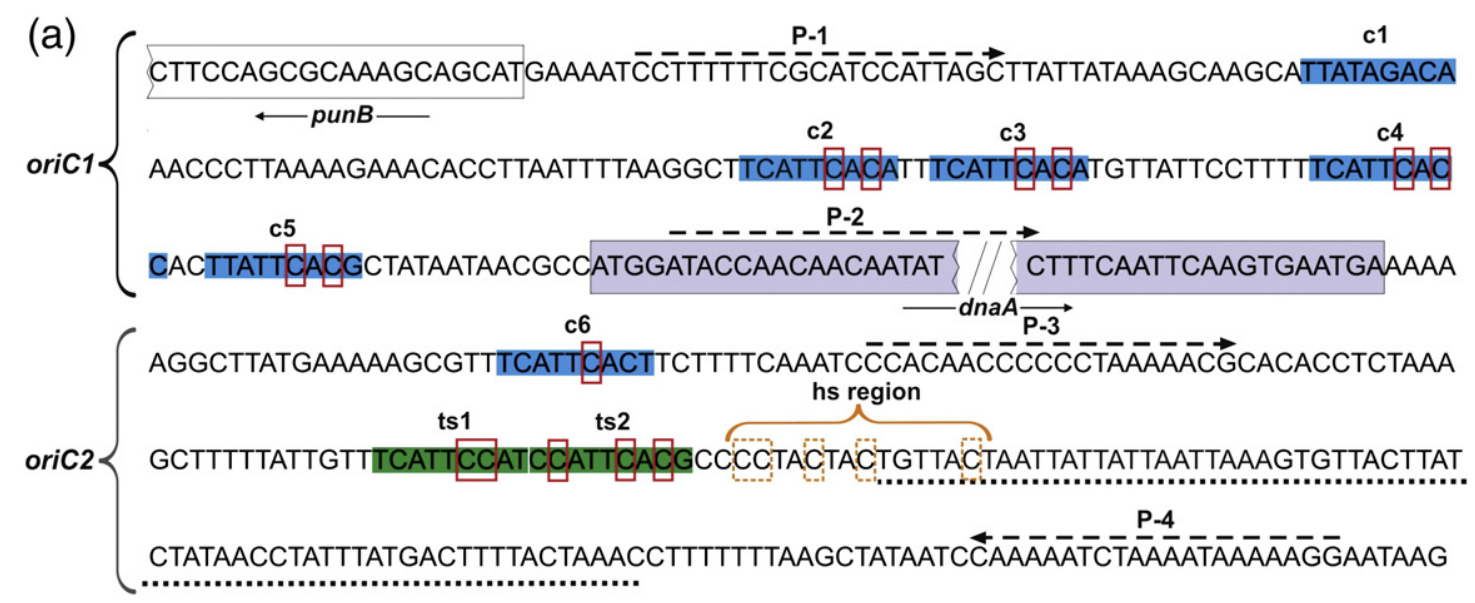

(b)
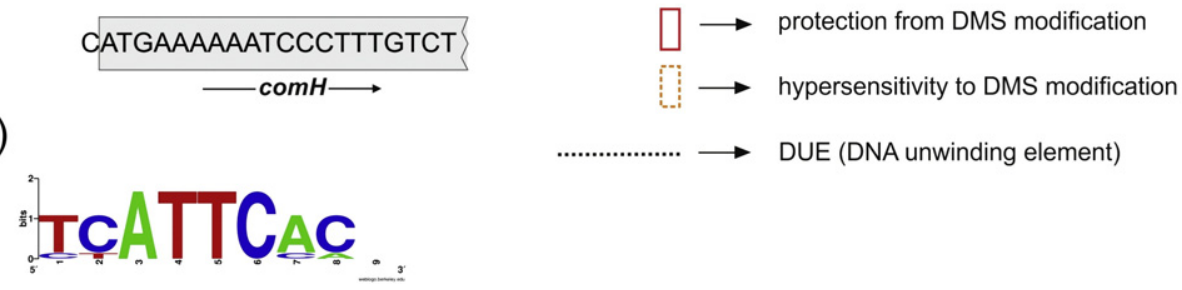

Fig. 3. (a) H. pylori bipartite oriC region. The results of DMS modification and subsequent PE analyses are presented. The positions of four of the five DnaA boxes at oriC1 were confirmed, and three novel DnaA boxes at oriC2 were identified. Additionally, a region partially overlapping the DUE that becomes hypersensitive to DMS upon protein binding (hs region) was detected. (b) The consensus sequence of $H$. pylori DnaA boxes on the basis of oriC1 and oriC2 boxes, with the exception of box c1. The logo was created with the WebLogo website (http://weblogo.berkeley.edu/; WebLogo: A Sequence Logo Generator) [37].

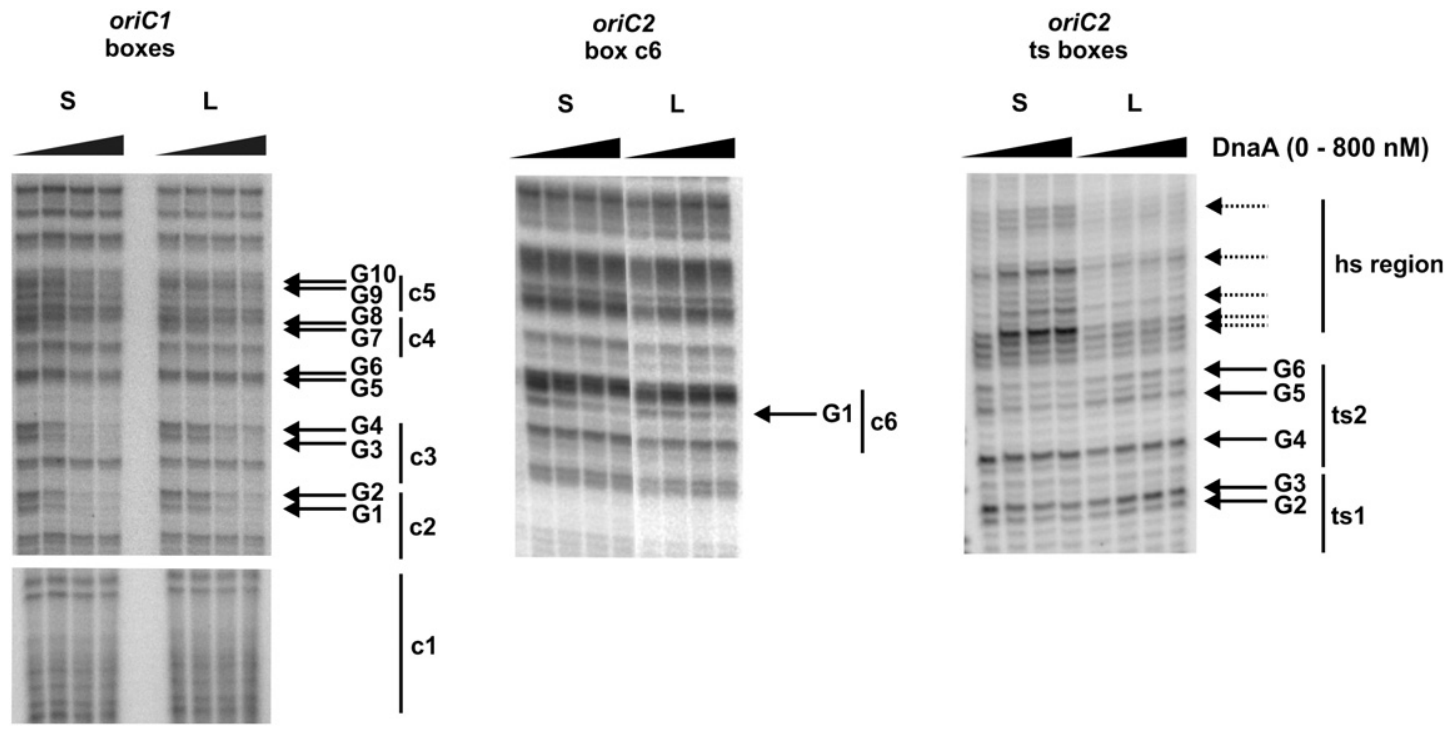

Fig. 4. Comparison of DnaA binding to supercoiled (S) and linear (L) oriC subregions. Plasmid pori1ori2 (supercoiled or linear) was incubated with the indicated amounts of the DnaA protein $(0,200,400$ and $800 \mathrm{nM})$, treated with DMS and then used as a substrate for PE analysis. ${ }^{32} \mathrm{P}$-labeled primers P-1 (oriC1), P-2 (oriC2 box c6) and P-3 (oriC2 ts boxes) are complementary to the lower strand. Continuous-line arrows indicate the nucleotides protected from DMS modification upon protein binding, and dotted-line arrows correspond to the nucleotides that become hypersensitive to DMS upon protein binding. The approximate positions of the identified boxes are marked next to the arrows. Please refer to Fig. 3 for details regarding the structure and sequence of the $H$. pylori oriC region. Densitometric plots supplementing this figure are presented in Fig. S2. 
upon binding of DnaA to supercoiled DNA (Fig. 4). The identification of ts DnaA boxes and the hs region, which are both susceptible to DnaA binding when supercoiled, establishes the importance of DNA superhelicity and, more generally, DNA topology, not only for DUE unwinding but also for binding of individual DnaA boxes by DnaA and orisome formation in H. pylori.

\section{Assessment of the role of individual oriC2 DnaA boxes in orisome formation}

Our results revealed that the $H$. pylori oriC2 subregion contains three DnaA boxes, two of which are bound only when supercoiled. To elucidate the role of individual oriC2 boxes in orisome formation and DNA unwinding, we analyzed DnaA binding to H. pylori oriC containing mutated DnaA boxes at oriC2 and comprehensively monitored orisome assembly by EM, DMS footprinting and analysis of open complex formation ( $\mathrm{P} 1$ nuclease assay). For these analyses, we prepared a series of plasmids derived from pori1ori2 in which sequences of DnaA boxes at oriC2 were substituted by direct mutagenesis (Fig. $5 \mathrm{a}$ and Table S1). The sequences of box c6, ts 1 and ts2 were changed to ACCGATTGC, GCCTAAAGT and ACTATGCTT, respectively. In total, four plasmids were constructed: ptsbox $2 \mathrm{~m}$, lacking box ts2; ptsbox $12 \mathrm{~m}$, devoid of both ts 1 and ts2 boxes; pcboxm, lacking box c6; and pdori2b, devoid of all three boxes. DMS footprinting analyses showed that these mutated boxes were not bound by DnaA, with the exception of plasmid ptsbox $12 \mathrm{~m}$, for which we detected a residual protection in the area of mutated boxes ts, particularly box ts1 (Fig. S4). We speculate that it reflects the role of a DNA tertiary structure of the oriC2 region in recruiting DnaA protein (see also below). In each of subsequent experiments, we used pori1ori2 as a control for DnaA interactions with wild-type oriC2.

EM analysis allowed us to estimate the influence of mutating each box on DnaA binding to each of the subregions and on the interactions between DnaAoriC1 and DnaA-oriC2 subcomplexes (Fig. $5 b$ and c). In the case of wild-type oriC2, $70 \%$ of the analyzed molecules were bound at oriC2 (note that this includes both molecules bound only at oriC2 and those bound simultaneously at oriC1 and oriC2, either forming or not forming a loop) and $26 \%$ were bound at oriC1 (Fig. 5c). The incidence of cross-interactions between the complexes was determined to be $23 \%$, showing that nearly all oriC1 subregions were joined to oriC2 by a DnaA protein core (Fig. 5c). Mutation of the ts2 box reduced DnaA binding to oriC2, as evidenced by an approximately $15 \%$ reduction in the number of DnaAbound plasmid molecules compared to the wild-type oriC2 (from $72 \%$ to $61 \%$ ) and a decrease in the number of DnaA-oriC2 subcomplexes from $70 \%$ to $58 \%$. Mutation of ts2 had virtually no effect on DnaA binding to oriC1 (25\%) or loop formation (22\%) (Fig. 5c). Further mutation of the topology-sensitive site by substitutions within the ts 1 box in the ptsbox $12 \mathrm{~m}$ plasmid additionally reduced the binding of DnaA to oriC2 by $10 \%$ (from $58 \%$ to $52 \%$ ), without changing the binding to oriC1 or loop formation (Fig. 5c). The mutation of a single box c6 on pcboxm reduced DnaA binding to oriC2 and oriC1 by $30 \%$ and $19 \%$, respectively. Mutation of this box additionally affected loop formation and reduced the number of cross-interacting suborigins by $35 \%$ (from $23 \%$ to 15\%) (Fig. 5c). This suggests that box c6 might be important for oriC1-oriC2 interaction via DnaA molecules bound to each of the suborigins. Mutation of all three boxes at oriC2 produced the most prominent effect on orisome formation; it reduced overall binding to pdori2b plasmid by $47 \%$ (from $72 \%$ to $38 \%$ ), interactions with oriC2 by $54 \%$ (from $70 \%$ to $32 \%$ ) and loop formation by $78 \%$ (from $23 \%$ to $5 \%$ ) (Fig. $5 \mathrm{c}$ ). Interestingly, nonspecific DnaA binding to the plasmid was increased (from 1\% to $7 \%$ ) in the triply mutated plasmid but binding to oriC1 was not changed. This is in agreement with the observation that oriC1 was poorly bound on a supercoiled plasmid, even in the absence of oriC2, and was presumably adjoined to the complex after oriC2 was bound [4]. In addition, the pdori2b plasmid retained a significant level of DnaAoriC2 interaction, suggesting that other structural features of oriC2 and/or additional DnaA binding sites, not detected by DMS footprint, are important for DnaA recognition and orisome formation in addition to the three identified DnaA boxes.

DMS footprinting was applied to analyze the influence of the respective mutated oriC elements on DnaA binding to the unmutated DnaA boxes and hypersensitivity to DMS modification (Fig. $5 \mathrm{~d}$ and Fig. S4). In this experiment, we also included a pori2 plasmid carrying the oriC2 subregion alone. We observed protection of the same nucleotides in pori2 as for pori1ori2 but detected no hs region (Fig. $5 d$ and Fig. S4a). We found that mutation of box c6 did not influence DnaA binding to ts boxes and vice versa (Fig. S4). Additionally, no clear changes in DMS hypersensitivity of the hs region were detected upon DnaA interactions following mutation of both ts boxes (Fig. 5d). Interestingly, mutation of a single box ts2 (plasmid ptsbox $2 \mathrm{~m}$ ) reduced hs effect. Furthermore, this mutation resulted in no detectable DnaA binding to box ts 1 , which suggests that efficient DnaA interaction with box ts 1 requires a cooperative interaction with box ts2 (Fig. S4a). It is also in agreement with the fact that box ts2 is bound stronger than ts 1 . Mutation of box $c 6$ had little effect on the hs region close to ts boxes (i.e., hypersensitivity was similar to that of wild-type oriC2), although residues more proximate to the DUE appeared to lose hypersensitivity to DMS as DnaA protein concentration increased (Fig. 5d). Mutation of all DnaA boxes in pdori2b plasmid resulted in the loss of 
(a) pori1ori2

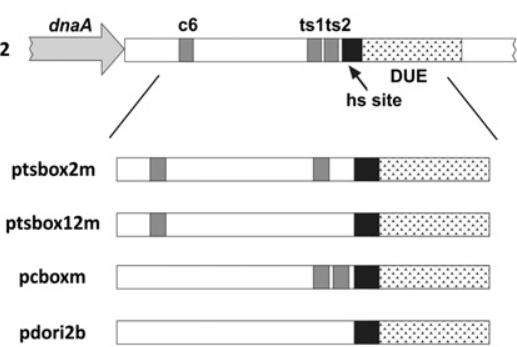

(b) supercoiled pori1ori2

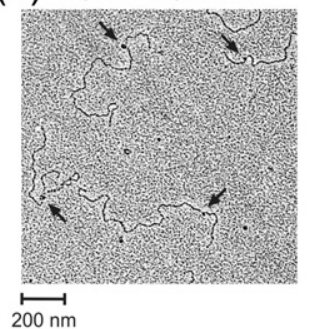

supercoiled ptsbox $12 \mathrm{~m}$
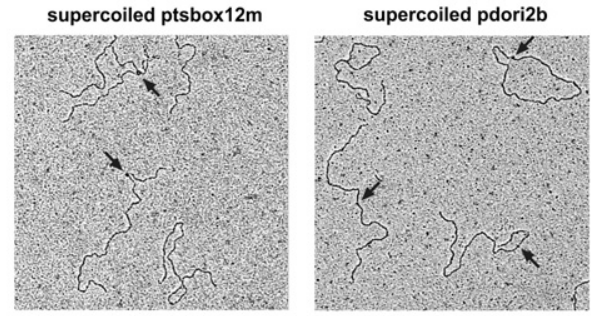

(c)

\begin{tabular}{|c|c|c|c|c|c|c|}
\hline \multirow[b]{2}{*}{ DNA } & \multirow[b]{2}{*}{$\begin{array}{c}\text { bound } \\
\text { molecules [\%] }\end{array}$} & \multicolumn{5}{|c|}{ molecules with particular region $(s)$ bound $[\%]$} \\
\hline & & oric2 & oric1 & $\begin{array}{c}\text { oric1 \& oric2 } \\
\text { with loop }\end{array}$ & $\begin{array}{c}\text { oric1 \& oric2 } \\
\text { no loop }\end{array}$ & other \\
\hline pori1ori2 & 72 & 70 & 26 & 23 & 2 & 1 \\
\hline ptsbox $2 m$ & 61 & 58 & 25 & 22 & 1 & 1 \\
\hline ptsbox12m & 56 & 52 & 28 & 24 & 0 & 3 \\
\hline pcboxm & 52 & 49 & 21 & 15 & 2 & 2 \\
\hline pdorizb & 38 & 32 & 25 & 5 & 10 & 7 \\
\hline
\end{tabular}

(d)

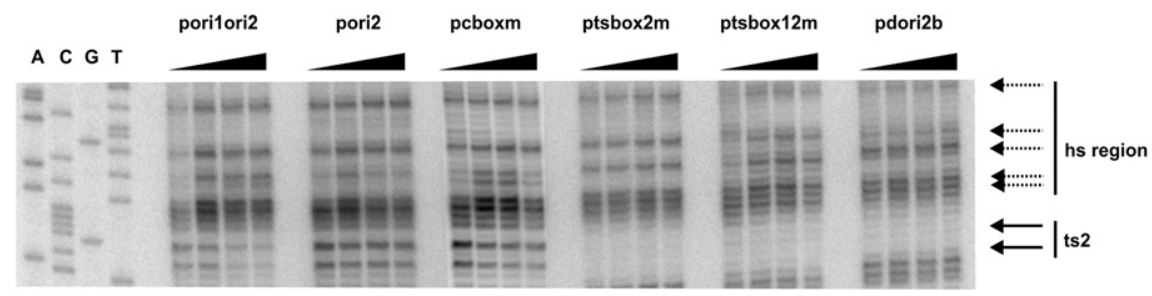

(e)
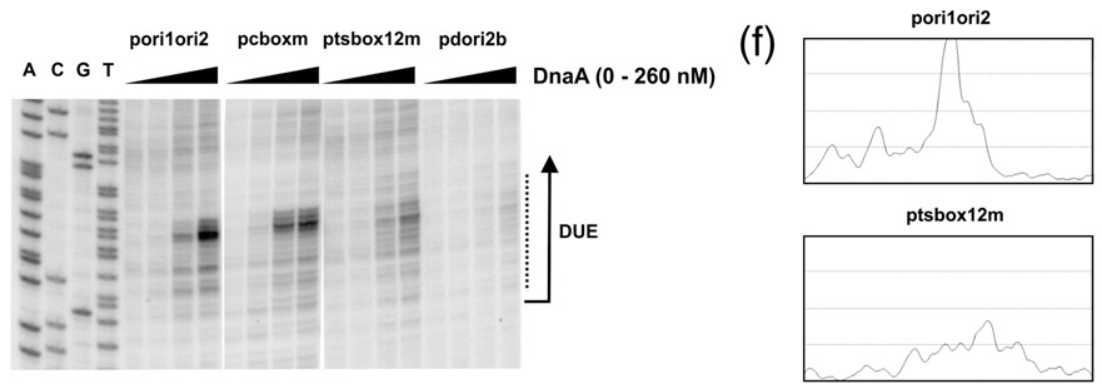

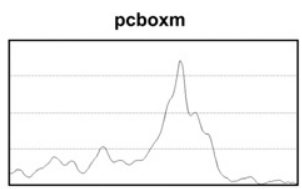

pdori2b

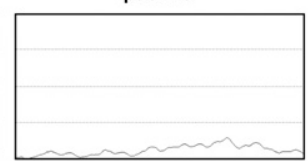

Fig. 5. Analysis of the role of individual oriC2 DnaA boxes in orisome formation. (a) Schematic representation of mutated plasmid constructs compared with the pori1ori2 plasmid containing a wild-type oriC region. (b) Representative images from an EM analysis of DnaA interaction with the indicated plasmids. (c) Statistical distribution and the level of DnaA binding to the indicated plasmids. The percentage of bound molecules and distribution of complexes were calculated based on an analysis of 150 molecules. Note that the percentage of molecules bound at oriC2 includes molecules bound at oriC2 alone and at both oriC1 and oriC2 (with or without a loop). Similarly, the percentage of molecules bound at oriC1 includes molecules bound at oriC1 alone and at both oriC1 and oriC2 (with or without a loop). (d) Analysis of region hypersensitive to DMS modification upon DnaA binding. The ${ }^{32} \mathrm{P}$-labeled $\mathrm{P}-3$ primer is complementary to the lower strand. Dotted-line arrows indicate the nucleotides that become hypersensitive to DMS upon protein binding to the wild-type oriC region (pori1ori2). These sites are diminished in pcboxm plasmid and are lost in pori2 and pdori2b plasmids. In case of ptsbox12m, they are unaffected. (e) DNA-unwinding analysis. Plasmid DNA was incubated with the indicated amounts of the DnaA protein $(0,65,130$ and $260 \mathrm{nM})$, treated with $\mathrm{P} 1$ nuclease and then used as a substrate for PE analysis. The ${ }^{32} \mathrm{P}$-labeled P-3 primer is complementary to the lower strand. (f) Densitometric plots obtained for the last lane (the highest DnaA protein concentration) for each plasmid in (e). The region, which was analyzed, is marked with a dotted line next to gel image.

hypersensitivity to DMS; that is, no difference was detected in the intensity of the bands arising from DMS treatment with and without DnaA (Fig. 5d). A similar result was observed with pori2. These results suggest that the long-distance interactions between DnaA complexes located at the bipartite origin are 
required to efficiently modify DNA structure in the oriC2 subregion (Fig. 5d and Fig. S4a) since the hs effect was gradually reduced together with a loss of loop structures (rank order of hypersensitivity: ptsbox $12 \mathrm{~m}>$ pcboxm $>$ pdori2b and pori2).

In the next experiment, we monitored the effects of the mutations of particular boxes on the unwinding of DUE upon orisome formation using a P1 nuclease assay (Fig. 5e and f and Fig. S3). The mutation of box 66 did not considerably reduce DUE unwinding of the pcboxm plasmid (Fig. 5e and f and Fig. S3). This corroborates the hypothesis that box c6 is mainly involved in long-distance interactions with oriC1, which are not necessary for DUE unwinding. The pori2 plasmid lacking oriC1 was unwound by DnaA at the DUE site, albeit to a much lesser extent than pori1ori2 [4]. However, in contrast to the case with the pori2 plasmid, the unwound DUE on the pcboxm plasmid did not decrease in size, which suggests a role for ts boxes, possibly together with interactions with oriC1, in stabilizing the width of the initiation bubble (Fig. 5e and Fig. S3a). In fact, mutation of ts boxes reduced the efficiency of DNA melting (i.e., less molecules were unwound at the same conditions as for the pori1ori2), but the size of the initiation bubble was not affected (Fig. 5e and f and Fig. S3). It is important to note that the observed difference in the unwinding level between pori1ori2 and ptsbox $12 \mathrm{~m}$ plasmids depends on the primer when monitored by PE reactions. Using primer P-3, we observed that the unwinding level was reduced by over $50 \%$ (Fig. $5 \mathrm{~d}$ and e), which is similar to the result obtained by restriction analysis (Fig. S3c). For primer P-4, the reduction was less substantial and was estimated for $25 \%$ (Fig. S3a and b). Additionally, the signals of PE reactions obtained by primer P-4 for all plasmids were less intense and more diffused when compared to that obtained by P-3 despite using the same templates for PE reactions and multiple experimental approaches. The difference is possibly caused by diverse digestion of upper and lower strands by P1 nuclease, which may result from variations in base composition and structure of single-stranded substrates [38]. Another possibility is a putative DnaA binding to SsDNA of the unwound DUE. DnaA might preferentially bind the upper strand of the DNA and protect it from P1 digestion, although such interaction has not been identified so far. Nevertheless, the results of Figs. $5 e$ and $f$ and $3 c$ taken together suggest that the ts boxes play more important role in DUE unwinding than box c6. Finally, mutation of all three DnaA boxes in oriC2 almost completely inhibited DUE unwinding (Fig. $5 e$ and $f$ and Fig. S3).

In the last experiment in this part of the study, we decided to determine if all three DnaA boxes are important for the $H$. pylori initiation of replication in vivo. A series of plasmids was constructed to enable mutation of box $c 6$ or both ts boxes and provide appropriate controls (Table S1 and Fig. S5). Plasmids pGEMc6mut and pGEMts12mut were prepared to change sequences of box c6 and ts boxes to ACCGATTGC and TCAAACGTAGGTATGGTC, respectively, and to introduce resistance cassette next to respective DnaA boxes, which facilitated the selection of recombinant colonies and ensured that $H$. pylori, capable to use unusually short (5 bps) homology arms for recombination [39,40], will not escape from the mutation by reducing homology arms (and thus introducing the resistance cassette but not mutated DnaA boxes into the chromosome). Plasmids pGEMc6c and pGEMts12c allowed for control transformations, which introduced resistance cassettes into exactly the same loci as in case of pGEMc6mut and pGEMts12mut plasmids, but did not change the sequences of boxes (Fig. S5). After transformation of H. pylori cells, we obtained recombinant colonies for the plasmid pGEMc6c and not pGEMc6mut, which proves that the inability to mutate box $c 6$ using plasmid pGEMc6mut was a result of its essential function in the cell and not the transformation problems (Fig. S5). On the other hand, we were unable to introduce resistance cassette between box $\mathrm{c} 6$ and ts boxes (plasmids pGEMts12mut and pGEMts12c). Thus, we could not determine if ts boxes are essential in vivo (Fig. S5). Nevertheless, such result suggests that the layout of particular elements of oriC2 is crucial for oriC2 function and changes of distance between boxes are likely to be lethal for the cell. Our results correspond well with the results of the previous study, which proved that it is not possible to delete the whole oriC2 subregion [4].

Collectively, our results show that ts 1 , ts 2 and $c 6$ boxes are important for oriC2 binding and the overall function of oriC2, especially DUE unwinding; c6 is probably mostly engaged in interactions with the DnaA-oriC1 subcomplex, whereas ts boxes are primarily important for DUE melting.

\section{Discussion}

Comprehensive studies of the initiation of replication in E. coli have shown that ordered binding of particular boxes by DnaA is important for proper DnaA oligomerization, orisome formation and, ultimately, orisome function. Indeed, the sequences and location of DnaA boxes at E. coli oriC affect their affinity for DnaA. Thus, the mode of DnaA assembly on oriC is partly regulated by oriC itself, which constitutes a molecular scaffold for DnaA oligomerization. Taking into account that the number and distribution of DnaA boxes in bacterial oriC regions vary greatly, it has been suggested that the mode of orisome formation is highly species specific $[6,22]$. Moreover, in some bacteria, the oriC region is subdivided into two parts, suggesting an even greater complexity in orisome structure and the regulation of its assembly. 
The $H$. pylori oriC was the first bipartite origin reported among numerous Gram-negative bacteria studied. Additionally, one part of the origin (oriC2) was shown to interact with DnaA exclusively as supercoiled DNA, which is highly unusual for bacteria in general [4]. In this work, we expand our understanding of the initiation of replication in $H$. pylori, determining the number and location of DnaA boxes in the oriC2 subregion, confirming their role in orisome formation and DNA unwinding and identifying the sequences involved in topology-dependent interactions.

\section{DnaA interaction with two of the novel DnaA boxes at oriC2 depends on DNA topology}

The fact that oriC2 is bound by DnaA only when DNA is supercoiled considerably limits the number of footprinting methods available for studying DnaAoriC2 interactions. Our initial experiments with DNase I and $\mathrm{Cu}-\mathrm{OP}$ were unsuccessful (data not shown) since both agents immediately damage the DNA and produce relaxation of the molecule, which presumably induces rapid dissociation of DnaA from DNA. We were also unable to obtain satisfactory results using UV footprinting since our regions of interest were poorly modified by UV radiation. Thus, to identify DnaA boxes in oriC2, we used DMS footprinting despite the fact that its detection specificity is limited to $G$ residues. Since all $H$. pylori DnaA boxes contain multiple $G$ residues solely on the lower strand (Fig. 3a), we used primers complementary to the lower strand in these analyses. Indeed, in the case of our analysis of DnaA binding to oriC1, we observed DnaA interactions with four of the five DnaA boxes identified in a previous study [32]; the 6th and 8th $\mathrm{G}$ residues were protected from DMS (Fig. 3a and Fig. S1). The box 11 was not protected from DMS modification, but DnaA binding to the single box $\mathrm{c} 1$ or, in fact, to any single box was found to be very weak in previous experiments $[32,33,41]$. We suspect that either the protein:DNA ratio we used was too low to detect this interaction with DMS or, more likely, box $\mathrm{c} 1$ is not a functional part of the $H$. pylori origin. The latter hypothesis is supported by the fact that box $c 1$ is the least conserved of all $H$. pylori DnaA boxes. In the case of the oriC2 region, we found three DnaA boxes located upstream of the DUE region (Figs. 2 and $3 a$ ). Similar to oriC1, the DnaA boxes at oriC2 are 9-mers, which is typical among bacterial origins (one exception is a Thermotoga maritima origin of replication, with 12-mer DnaA boxes [42]). All DnaA boxes at oriC2 have the same orientation as oriC1 boxes; thus, all eight $H$. pylori boxes are orientated in the same direction. This feature is unique among experimentally confirmed bacterial origins, whether composed of a single DnaA box cluster, as in E. coli, or bipartite, as in B. subtilis, which always contain boxes with both orientations $[3,9]$. The role of such a distribution of boxes in orisome formation in $\mathrm{H}$. pylori oriC is unknown, but together with the observed bipartite structure and topology-dependent DnaA interactions, it clearly illustrates that this region is atypical among known bacterial origins. The sequence of box c6 corresponds with the consensus sequence of boxes at oriC1 $(\mathrm{T}(\mathrm{C} /$ T)ATTCACN) that, in turn, corresponds with the E. coli consensus sequence (TTATNCACA). ts boxes, on the other hand, have sequences that differ from the consensus of c-boxes by one or two nucleotides. On the basis of the sequences of DnaA boxes in both parts of the $H$. pylori origin, we propose that the consensus sequence of DnaA boxes in $H$. pylori (with the exception of box c1) is TCATTCACN (Fig. 3b). DnaA affinity to ts boxes depends on DNA topology. Since the sequences of ts boxes are not substantially different from those of other $H$. pylori boxes, one intriguing explanation for this phenomenon is the presence of a unique DNA tertiary structure that reflects the sequence of all or a part of the oriC2 region. The biological role of this mode of binding requires further study, but we think it likely that it constitutes another level of regulation of replication initiation. To date, only one factor affecting initiation of replication (HobA) has been identified in $H$. pylori [34]. The $H$. pylori genome does not encode proteins homologues to Fis or IHF, which regulate orisome formation in E. coli. Furthermore, H. pylori does not appear to control initiation frequency by changing intracellular levels of DnaA (A. Zawilak-Pawlik, unpublished results). Accordingly, it is likely that, in $H$. pylori, the DNA topology of the oriC region serves to regulate DnaA affinity. This manner of initiation complex formation resembles initiator-origin interactions in archaea, simple eukaryotes and, most importantly, metazoans, which are reported to depend less on DNA sequence and more on DNA tertiary structure [43-45]. A continuum of origin binding mechanisms has been suggested, and bacteria have been proposed to depend strictly on DNA sequence, not topology [44]. The mode of orisome formation in $H$. pylori may indicate that topology-dependent origin recognition is common among organisms in all three domains of life. However, no such dependence has been reported for $E$. colior $B$. subtilis $[10,46]$. Since the $H$. pylori genome encodes few regulatory proteins [47], it is possible, and in fact has already been suggested [48], that DNA topology constitutes a regulatory factor in $H$. pylori with a much more prominent role in this species than in other bacteria. It is also plausible that a similar phenomenon might be important for regulating DNA-associated processes in other highly specialized microorganisms with reduced genomes.

\section{All three oriC2 DnaA boxes are required for efficient orisome formation}

After identification and initial characterization of DnaA boxes in oriC2, we analyzed their role in 
orisome formation and DNA unwinding (Fig. 5). We observed that the lack of single box ts2 or both ts boxes had a moderate influence on DnaA binding to oriC2, reducing it by $\sim 25 \%$ compared with wild-type oriC2. In contrast, mutating these boxes did not influence DnaA binding to oriC1. Interestingly, mutation of the single box c6 significantly inhibited loop formation between oriC1 and oriC2, whereas the lack of both ts boxes did not change the incidence of loops. On the other hand, mutation of the single box c6 did not considerably affect DNA unwinding, but mutation of ts boxes reduced unwinding level by at least $25 \%$. Collectively, these observations suggest that box $c 6$ is sufficient to support long-distance interactions with oriC1 even in the absence of ts boxes, whereas ts boxes effectively support unwinding in the absence of box c6. Additionally, the lack of box c6 reduced the hypersensitivity to DMS in the region adjacent to the DUE. Finally, mutagenesis of all three boxes inhibited DnaA binding to oriC2 by approximately $50 \%$, disrupted loop formation and abolished unwinding. The supplementary role of box c6 in DNA unwinding is in agreement with the fact that oriC2 is susceptible to limited DnaA-dependent DNA melting in the absence of oriC1 [4] and, consequently, in the absence of long-distance DnaA interactions, which are mainly supported by box c6. Additionally, box c6 is essential in vivo, which corroborates the hypothesis that efficient loop formation between oriC1 and oriC2 is crucial for the initiation mechanism (Fig. S5). The DUE-proximate position of ts boxes (10 bp from the DUE) may explain their influence on the unwinding reaction. The $E$. coli high-affinity DnaA box $\mathrm{R} 1$ is also located close (12 bp) to the DUE and was shown to be indispensible for DNA unwinding and to assist in ssDNA binding in vitro $[18,49]$. Though it has been recently proved that box $\mathrm{R} 1$ is dispensable in vivo [50], the reduced initiation frequency and asynchrony of the initiation of replication in R1-box-mutated strains corroborates previous results indicating that this box is important for replication of the E. coli chromosome [51]. The results of our work further support a model in which a box (or boxes) located in the vicinity of the DUE are important for DNA unwinding. For most of the plasmids analyzed in this work (except pori2 and pdori2b), we observed a hypersensitivity to DMS in the region adjacent to the DUE. In our opinion, this phenomenon probably indicates alterations in DNA structure introduced by orisome complex assembly and loop formation. At present stage of the study, it is impossible to better characterize the hs effect and connect it with particular changes to DNA structure (e.g., DNA bending/looping), since the occurrence of hs effect does not correlate with the putative DNA bending observed on some EM photographs (this study and Ref. [4]). Higher-resolution methods are required to reliably analyze the DNA bending properties of a DnaA oligomer.
The results presented here suggest that all three boxes are required for efficient DnaA binding to oriC2 and origin activity, but the lack of any single element (box c6 or ts boxes) can be overcome to some extent, at least in vitro. Interestingly, it was shown recently that, in $E$. coli, no individual highaffinity DnaA box is absolutely essential for oriC function in vitro or in vivo [50]. The reason why the lack of all three oriC2 DnaA boxes did not abolish DnaA binding may be that the oriC2 tertiary structure per se is, at least to some extent, recognized by DnaA. However, it should be noted that, without the specificity and precision guaranteed by the presence of DnaA boxes, DnaA does not perform its functions properly since in such case DNA melting is severely inhibited. It is also likely that $H$. pylori oriC contains other sequences that possess a very low affinity for DnaA or are recognized by DnaA only in certain conditions. It is noteworthy that, in the last 10 years, the $E$. coli oriC region, which had been previously thought to contain five DnaA boxes, has been shown to have a much more complicated structure, including the presence of numerous low-affinity DnaA boxes recognized only by ATP-DnaA $[8,9]$. It is possible that we were unable to detect such weak interactions owing to the limitations of the DMS footprinting method (see above). In the future, we plan to continue our characterization of the $H$. pylori orisome formation mechanism and initiation process, which may lead to the identification of additional steps in the process and novel sequences recognized by DnaA.

\section{DnaA molecules bound to individual origin subregions serve different functions in replication initiation}

Many bacterial origins are divided into two parts [52]. One of the best characterized examples is $B$. subtilis oriC, which is composed of three DnaA box clusters: inc $A$ and incB, located upstream of the $d n a A$ gene, and incC together with the DUE, located downstream $[3,46,53]$. The interaction of DnaA with these three clusters results in the formation of a loop structure, as occurs similarly in H. pylori [46]. Why oriC regions are split and what exact role the loop structures play is still unknown [4,52], especially given that the DNA-unwinding activity of the DUE proximal subregion is usually retained following removal of the DUE distal subregion. In the case of $B$. subtilis, it has been shown that the DUE is melted in the absence of inc $A$ and incB DnaA box clusters [46], whereas oriC2 in H. pylori is sufficient for DnaA-dependent DUE unwinding, although to a limited extent [4]. The bipartite structure may have a regulatory function in DNA unwinding, as was suggested for incA/incB DnaA boxes, which might serve as replication enhancers for DUE melting downstream of $d n a A$ [46]. This structure might also be connected to different functions of DnaA oligomers formed on 
particular subregions. For example, it may be necessary for separate assembly of the two replisomes onto leading and lagging DNA strands [52]. Interestingly, a dual structure of the $E$. coli orisome has been recently proposed [26,27]. DnaA molecules bound to DnaA boxes situated close to the DUE were shown to be primarily involved in DUE unwinding, single-stranded DUE binding and helicase binding and loading, whereas DnaA molecules bound to boxes from R2 to R4 were proposed to have an auxiliary role in helicase binding and loading. Additionally, this right half-part of oriC was shown to be redundant in slow-growing cells [28]. It seems likely that the $H$. pylori orisome is also functionally divided: DnaA molecules interacting with ts boxes might be responsible for DNA unwinding and stabilization of nascent ssDNA (i.e., single-stranded DUE), and the remaining DnaA molecules, bound to boxes from c1 to $c 6$ and creating a DNA loop, might support unwinding and be involved in successive steps of initiation (e.g., helicase loading). Interestingly, the lack of oriC1 results in a limited number of unwound molecules in vitro and a significant decrease in the size of the initiation bubble. Thus, oriC1 boxes, together with box c6, might be involved in regulating the proper positioning of the
$H$. pylori replicative helicase. This function might be especially important because no helicase loader has been identified in $H$. pylori, and the helicase has been suggested to function via a self-loading mechanism [54]. Similar differentiation of the function of DnaA molecules bound to different parts of the origin may also apply to other bacteria.

On the basis of the results of this and previous works, we propose a model of the assembly of the initiation complex in $H$. pylori (Fig. 6). According to this model, DnaA interacts with each of the suborigins, ultimately linking them together through a DnaA protein core. DnaA binding to oriC1 is enhanced by HobA, whereas DnaA binding to oriC2 is conditioned on its negative superhelicity; the role of HobA in DnaA interaction with oriC2 is still unknown, similarly as the extent to which changes to DNA topology control the initiation mechanism and how these changes may be connected with the dynamics of the entire chromosome. Upon crossinteraction of origin subcomplexes, the final initiation complex is assembled, leading to DUE unwinding. DnaA molecules situated at double-stranded DnaA boxes (i.e., ts boxes) might stabilize ssDNA, although such interaction has not been reported

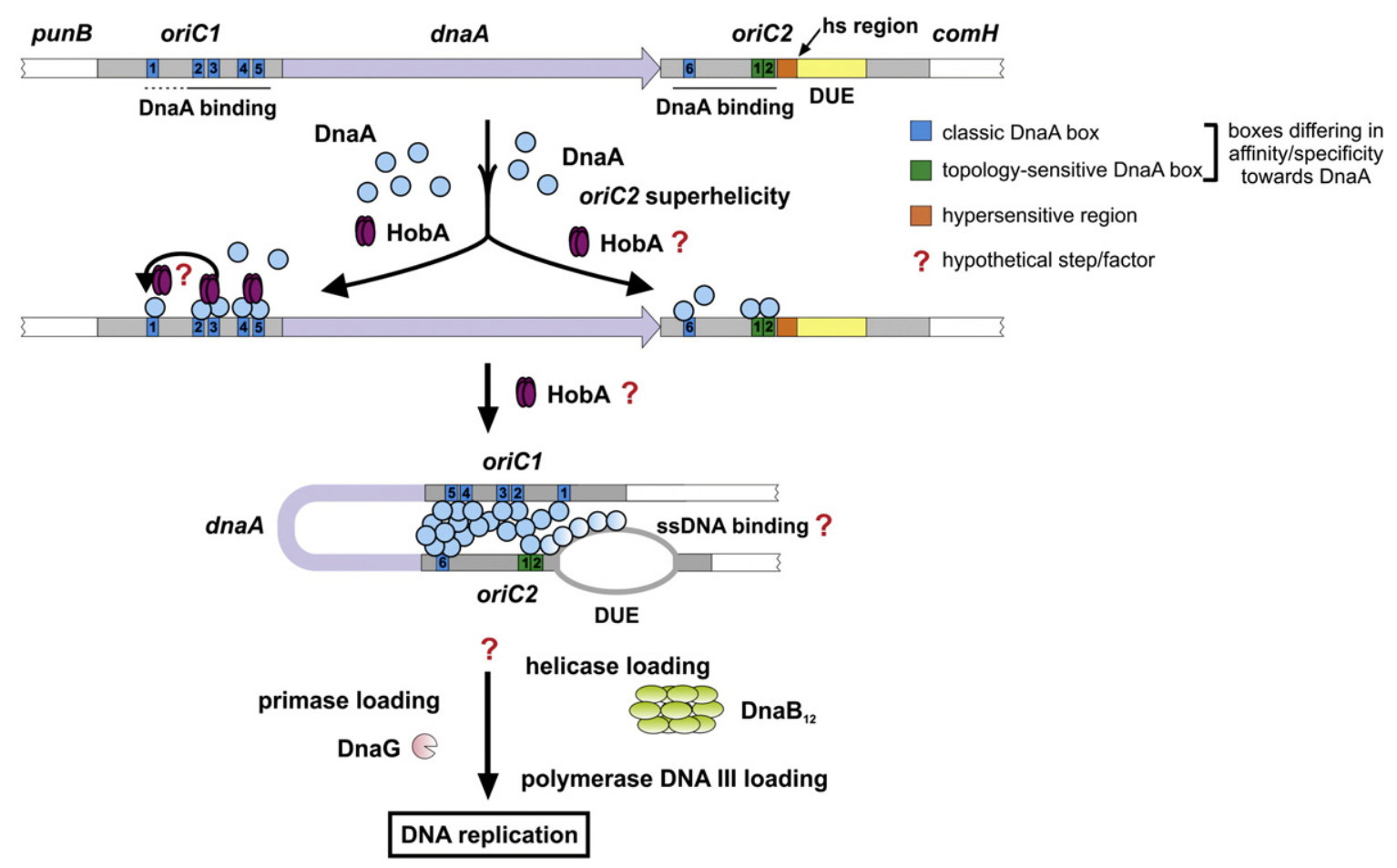

Fig. 6. Model of the initiation of chromosome replication in $H$. pylori based on this and previous works. DnaA binding to the bipartite origin is facilitated by the HobA protein at oriC1 and by DNA topology at oriC2. The role of HobA in orisome formation at oriC2 is unknown. DnaA molecules bound at both oriC subregions cross-interact, leading to looping of the $d n a A$ gene, final assembly of the initiation complex and DNA unwinding at oriC2. The initiation bubble is formed and ssDNA might be bound by DnaA molecules anchored at ts boxes, similarly as in $E$. coli. In the subsequent steps, DnaB helicase and other proteins of the replisome are loaded into it through an as yet unknown mechanism. 
so far. The initiation bubble is formed, and DnaB helicase and other proteins of the replisome are loaded into it through a mechanism that has yet to be discovered.

\section{Materials and Methods}

\section{Materials and culture conditions}

The plasmids, proteins and nucleotides used in this work are listed in Table S1. The oligonucleotide sequences are presented in Table S2. E. coliwas grown at $37^{\circ} \mathrm{C}$ on solid or liquid Luria-Bertani medium, supplemented with $100 \mu \mathrm{g} / \mathrm{ml}$ ampicillin as necessary. Plasmids for all experiments were purified using a Plasmid Midi Kit (Qiagen).

\section{In vitro mutagenesis}

In order to analyze the influence of particular DnaA boxes on DnaA binding to oriC2 and subsequent DNA unwinding, we prepared a series of plasmids with mutagenized DnaA boxes (Table S1). Plasmid ptsbox2m (Fig. 3) was constructed using a two-step approach. First, two PCR products were obtained with primer pairs $\mathrm{P}-5 / \mathrm{P}-8$ and $\mathrm{P}-6 / \mathrm{P}-7$. The resulting fragments were used as templates in a fusion PCR with primers P-5 and P-6. The product was digested with EcoRI/Pstl and cloned into the pori1ori2 plasmid digested with the same restriction enzymes. Plasmids ptsbox $12 \mathrm{~m}$, pcboxm and pdori2b were prepared in a similar way, substituting the following primers for P-7 and P-8:P-9/ $\mathrm{P}-10$ for ptsbox12m, P-11/P-12 for pcboxm, and P-11/P-12 for pdori2b. The final fusion PCR products were cloned into ptsbox $2 \mathrm{~m}$ to obtain ptsbox $12 \mathrm{~m}$, pori1ori2 to obtain pcboxm and ptsbox $12 \mathrm{~m}$ to obtain pdori2b.

\section{In vivo mutagenesis}

In order to mutate box c6 or both ts boxes, we prepared a series of plasmids bearing DNA fragments, which allowed for homologous recombination with desired $H$. pylori chromosomal regions. Plasmids were constructed using following approach. First, four pairs of $P C R$ products were obtained with following primers: $P-13 / P-16$ and $P-14 / P-15 ; P-13 / P-18$ and P-14/P-17; P-13/P-20 and P-14/P-19; P-13/P-22 and P-14/ $\mathrm{P}-21$. The resulting pairs of $\mathrm{PCR}$ products were subsequently used as templates in a fusion $\mathrm{PCR}$ with primers $\mathrm{P}-13$ and $\mathrm{P}-14$ to give four DNA fragments containing: homologous regions (ca 250 bp) upstream and downstream of oriC2, BamHI/Pstl restriction sites for cloning of a resistance gene and mutated boxes c6 or ts if required. The four DNA fragments were afterwards digested with Pael/Sall restriction enzymes and cloned into pGEM-T Easy vector digested with the same enzymes. The resulting pGEM-T Easy derivatives were digested with BamHI/Pstl restriction enzymes and ligated with kanamycin resistance gene digested from $x x x$ vector using the same enzymes. The following plasmids were obtained: pGEMc6mut and pGEMts12mut for the mutation of boxes $c 6$ and ts, respectively, and pGEMc6c and pGEMts12c, which served as controls of respective transformations (Table S1 and Fig. S5).

\section{DNA modification with DMS}

In vitro DNA treatment was performed as described by Sasse-Dwight and Gralla [55]. Reaction mixtures (50 $\mu \mathrm{l}$ ) contained 25 mM Hepes-KOH (pH 7.6), 12\% (v/v) glycerol, $1 \mathrm{mM} \mathrm{CaCl} 2,0.2 \mathrm{mM}$ ethylenediaminetetraacetic acid, $5 \mathrm{mM}$ ATP, $0.1 \mathrm{mg} / \mathrm{ml}$ bovine serum albumin, $2 \mu \mathrm{g}$ of plasmid DNA (10 nM) and H. pylori DnaA protein (200, 400 and $800 \mathrm{nM})$. After incubation at $30{ }^{\circ} \mathrm{C}$ for $10 \mathrm{~min}, 3.6 \mu \mathrm{l}$ of $150 \mathrm{mM} \mathrm{DMS}$ (Sigma) was added to a final concentration of $10 \mathrm{mM}$, and the incubation was continued for $5 \mathrm{~min}$. The reaction was quenched with the addition of $100 \mu \mathrm{l}$ of cold Stop Buffer (3 M ammonium acetate, 1 M 2-mercaptoethanol and $20 \mathrm{mM}$ ethylenediaminetetraacetic acid). Samples were precipitated with cold ethanol, dried, dissolved in $100 \mu \mathrm{l}$ of $1 \mathrm{M}$ piperidine and incubated at $90^{\circ} \mathrm{C}$ for $30 \mathrm{~min}$. DNA was purified by gel filtration on Sephacryl S500 (Sigma) spin columns equilibrated in molecular-grade water. The DMS modification pattern was monitored by PE analysis.

\section{P1 nuclease assay}

The P1 nuclease assay protocol was similar to that previously described [4]. Reaction mixtures $(15 \mu \mathrm{l})$ contained $200 \mathrm{ng}(5 \mathrm{nM})$ of the indicated plasmid and H. pylori DnaA (65, 130 and $260 \mathrm{nM})$. P1 digestion results were monitored by $\mathrm{PE}$ analysis or restriction enzyme digestion. In latter case, the whole DNA after P1 treatment and purification was digested by EcoRI, loaded on a $1 \%$ agarose gel and separated. After ethidium bromide staining, we scanned the gels with a Typhoon 8600 Variable Mode Imager (GE Healthcare). Images were analyzed with ImageJ software ${ }^{\dagger}$ [56]. Representative scans and densitometric plots are shown in the figures.

\section{PE analysis}

The PE method was used to monitor the results of DMS modification and P1 nuclease assays. For a single PE reaction [55], 0.3 units of Taq DNA polymerase (Thermo Scientific), $20 \mathrm{fmol}$ of DNA template and $350 \mathrm{fmol}{ }^{32} \mathrm{P}$-labeled primer were used. After PE (30 cycles of $30 \mathrm{~s}$ at $95^{\circ} \mathrm{C}, 30 \mathrm{~s}$ at $55^{\circ} \mathrm{C}$ and $60 \mathrm{~s}$ at $72{ }^{\circ} \mathrm{C}$ ), samples were separated on an $8 \%$ polyacrylamide gel under denaturing conditions and scanned with a Typhoon 8600 Variable Mode Imager (GE Healthcare). Images were analyzed with ImageJ software $^{1}$ [56]. Representative scans and densitometric plots are shown in the figures.

\section{EM analysis}

EM was performed exactly as previously described [4]. Reaction mixtures $(20 \mu \mathrm{l})$ contained $60 \mathrm{ng}(1.5 \mathrm{nM})$ of the indicated plasmid and $40 \mathrm{nM} \mathrm{H}$. pylori DnaA protein. After glutaraldehyde titration with glycylglycine, all samples were digested with Scal restriction enzyme. Nucleoprotein complexes were analyzed using a Philips CM100 electron microscope (FEI, Hillsboro, USA) equipped with a Fastscan CCD camera (TVIPS, Gautung, Germany). The positions of the proteins bound to DNA were measured on $35 \mathrm{~mm}$ negatives using a LM4 digitizer (Brühl, Nürnberg, Germany). 


\section{Acknowledgements}

We thank Christoph Weigel for critical comments concerning the manuscript. This work was supported by a research grant from the National Science Center (Preludium Project DEC-2012/05/N/NZ1/ 00021).

\section{Appendix A. Supplementary data}

Supplementary data to this article can be found online at http://dx.doi.org/10.1016/j.jmb.2014.05.018.

Received 19 February 2014; Received in revised form 15 May 2014; Accepted 16 May 2014

Available online 24 May 2014

Keywords:

DNA replication; orisome formation;

DNA topology;

DNA sequence;

oriC

†http://imagej.nih.gov/ij

Abbreviations used:

DUE, DNA-unwinding element; EM, electron microscopy; $\mathrm{PE}$, primer extension; ssDNA, single-stranded DNA.

\section{References}

[1] Nielsen O, Løbner-Olesen A. Once in a lifetime: strategies for preventing re-replication in prokaryotic and eukaryotic cells. EMBO Rep 2008;9:151-6.

[2] Kawakami H, Katayama T. DnaA, ORC, and Cdc6: similarity beyond the domains of life and diversity. Biochem Cell Biol Biochim Biol Cell 2010;88:49-62.

[3] Moriya S, Fukuoka T, Ogasawara N, Yoshikawa H. Regulation of initiation of the chromosomal replication by DnaA-boxes in the origin region of the Bacillus subtilis chromosome. EMBO J 1988;7:2911-7.

[4] Donczew R, Lurz R, Zakrzewska-Czerwinska J, ZawilakPawlik A. Helicobacter pylori oriC — the first bipartite origin of chromosome replication in Gram-negative bacteria. Nucleic Acids Res 2012;40:9647-60.

[5] Rajewska M, Wegrzyn K, Konieczny I. AT-rich region and repeated sequences - the essential elements of replication origins of bacterial replicons. FEMS Microbiol Rev 2012;36:408-34.

[6] Gao F, Luo H, Zhang C-T. DoriC 5.0: an updated database of oriC regions in both bacterial and archaeal genomes. Nucleic Acids Res 2013;41:D90-3.

[7] Kaguni JM. DnaA: controlling the initiation of bacterial DNA replication and more. Annu Rev Microbiol 2006;60:351-75.

[8] Ozaki S, Katayama T. DnaA structure, function, and dynamics in the initiation at the chromosomal origin. Plasmid 2009;62:71-82.

[9] Leonard AC, Grimwade JE. Regulation of DnaA assembly and activity: taking directions from the genome. Annu Rev Microbiol 2011:65:19-35.

[10] Weigel C, Schmidt A, Rückert B, Lurz R, Messer W. DnaA protein binding to individual DnaA boxes in the Escherichia coli replication origin, oriC. EMBO J 1997;16:6574-83.

[11] Margulies C, Kaguni JM. Ordered and sequential binding of DnaA protein to oriC, the chromosomal origin of Escherichia coli. J Biol Chem 1996;271:17035-40.

[12] Schaper S, Messer W. Interaction of the initiator protein DnaA of Escherichia coli with its DNA target. J Biol Chem 1995;270:17622-6.

[13] Grimwade JE, Torgue JJ, McGarry KC, Rozgaja T, Enloe ST, Leonard AC. Mutational analysis reveals Escherichia coli oriC interacts with both DnaA-ATP and DnaA-ADP during pre-RC assembly. Mol Microbiol 2007;66:428-39.

[14] Kawakami H, Keyamura K, Katayama T. Formation of an ATP-DnaA-specific initiation complex requires DnaA Arginine 285 , a conserved motif in the AAA + protein family. J Biol Chem 2005;280:27420-30.

[15] Ozaki S, Kawakami H, Nakamura K, Fujikawa N, Kagawa W, Park SY, et al. A common mechanism for the ATP-DnaAdependent formation of open complexes at the replication origin. J Biol Chem 2008;283:8351-62.

[16] Ryan VT, Grimwade JE, Nievera CJ, Leonard AC. IHF and HU stimulate assembly of pre-replication complexes at Escherichia coli oriC by two different mechanisms. Mol Microbiol 2002;46:113-24.

[17] Hansen FG, Christensen BB, Atlung T. Sequence characteristics required for cooperative binding and efficient in vivo titration of the replication initiator protein DnaA in E. coli. J Mol Biol 2007;367:942-52.

[18] Speck C, Messer W. Mechanism of origin unwinding: sequential binding of DnaA to double- and single-stranded DNA. EMBO J 2001;20:1469-76.

[19] Keyamura K, Fujikawa N, Ishida T, Ozaki S, Su'etsugu M, Fujimitsu K, et al. The interaction of DiaA and DnaA regulates the replication cycle in $E$. coli by directly promoting ATP DnaAspecific initiation complexes. Genes Dev 2007;21:2083-99.

[20] Rozgaja TA, Grimwade JE, Iqbal M, Czerwonka C, Vora M, Leonard AC. Two oppositely oriented arrays of low-affinity recognition sites in oriC guide progressive binding of DnaA during Escherichia coli pre-RC assembly. Mol Microbiol 2011;82:475-88.

[21] Miller DT, Grimwade JE, Betteridge T, Rozgaja T, Torgue JJ, Leonard AC. Bacterial origin recognition complexes direct assembly of higher-order DnaA oligomeric structures. Proc Natl Acad Sci USA 2009;106:18479-84.

[22] Leonard AC, Grimwade JE. Regulating DnaA complex assembly: it is time to fill the gaps. Curr Opin Microbiol 2010;13:766-72.

[23] Zorman S, Seitz H, Sclavi B, Strick TR. Topological characterization of the DnaA-oriC complex using single-molecule nanomanipuation. Nucleic Acids Res 2012;40:7375-83.

[24] Grimwade JE, Ryan VT, Leonard AC. IHF redistributes bound initiator protein, DnaA, on supercoiled oriC of Escherichia coli. Mol Microbiol 2000;35:835-44.

[25] Erzberger JP, Mott ML, Berger JM. Structural basis for ATPdependent DnaA assembly and replication-origin remodeling. Nat Struct Mol Biol 2006;13:676-83.

[26] Ozaki S, Noguchi Y, Hayashi Y, Miyazaki E, Katayama T. Differentiation of the DnaA-oriC subcomplex for DNA 
unwinding in a replication initiation complex. $\mathrm{J}$ Biol Chem 2012;287:37458-71.

[27] Keyamura K, Abe Y, Higashi M, Ueda T, Katayama T. DiaA dynamics are coupled with changes in initial origin complexes leading to helicase loading. J Biol Chem 2009;284:25038-50.

[28] Stepankiw N, Kaidow A, Boye E, Bates D. The right half of the Escherichia coli replication origin is not essential for viability, but facilitates multi-forked replication. Mol Microbiol 2009;74:467-79.

[29] Zakrzewska-Czerwińska J, Jakimowicz D, Zawilak-Pawlik A, Messer W. Regulation of the initiation of chromosomal replication in bacteria. FEMS Microbiol Rev 2007;31:378-87.

[30] Katayama T, Ozaki S, Keyamura K, Fujimitsu K. Regulation of the replication cycle: conserved and diverse regulatory systems for DnaA and oriC. Nat Rev Microbiol 2010;8:163-70.

[31] Saxena R, Fingland N, Patil D, Sharma AK, Crooke E. Crosstalk between DnaA protein, the initiator of Escherichia colichromosomal replication, and acidic phospholipids present in bacterial membranes. Int J Mol Sci 2013;14:8517-37.

[32] Zawilak A, Cebrat S, Mackiewicz P, Król-Hulewicz A, Jakimowicz D, Messer $W$, et al. Identification of a putative chromosomal replication origin from Helicobacter pylori and its interaction with the initiator protein DnaA. Nucleic Acids Res 2001;29:2251-9.

[33] Zawilak A, Durrant MC, Jakimowicz P, Backert S, Zakrzewska-Czerwińska J. DNA binding specificity of the replication initiator protein DnaA from Helicobacter pylori. J Mol Biol 2003;334:933-47.

[34] Zawilak-Pawlik A, Kois A, Stingl K, Boneca IG, Skrobuk P, Piotr J, et al. HobA-a novel protein involved in initiation of chromosomal replication in Helicobacter pylori. Mol Microbiol 2007;65:979-94.

[35] Zawilak-Pawlik A, Donczew R, Szafrański S, Mackiewicz P, Terradot L, Zakrzewska-Czerwińska J. DiaA/HobA and DnaA: a pair of proteins co-evolved to cooperate during bacterial orisome assembly. J Mol Biol 2011;408:238-51.

[36] Tomb JF, White O, Kerlavage AR, Clayton RA, Sutton GG, Fleischmann $\mathrm{RD}$, et al. The complete genome sequence of the gastric pathogen Helicobacter pylori. Nature 1997;388:539-47.

[37] Crooks GE, Hon G, Chandonia J-M, Brenner SE. WebLogo: a sequence logo generator. Genome Res 2004;14:1188-90.

[38] Box HC, Budzinski EE, Evans MS, French JB, Maccubbin $A E$. The differential lysis of phosphoester bonds by nuclease P1. Biochim Biophys Acta 1993;1161:291-4.

[39] Levine SM, Lin EA, Emara W, Kang J, DiBenedetto M, Ando $T$, et al. Plastic cells and populations: DNA substrate characteristics in Helicobacter pylori transformation define a flexible but conservative system for genomic variation. FASEB J 2007;21:3458-67.

[40] Lin EA, Zhang XS, Levine SM, Gill SR, Falush D, Blaser MJ. Natural transformation of Helicobacter pylori involves the integration of short DNA fragments interrupted by gaps of variable size. PLoS Pathog 2009;5:e1000337.
[41] Zawilak-Pawlik A, Kois A, Majka J, Jakimowicz D, SmulczykKrawczyszyn A, Messer W, et al. Architecture of bacterial replication initiation complexes: orisomes from four unrelated bacteria. Biochem J 2005;389:471-81.

[42] Lopez P, Forterre P, le Guyader H, Philippe H. Origin of replication of Thermotoga maritima. Trends Genet TIG 2000;16:59-60.

[43] Remus D, Beall EL, Botchan MR. DNA topology, not DNA sequence, is a critical determinant for Drosophila ORC-DNA binding. EMBO J 2004;23:897-907.

[44] Dueber EC, Costa A, Corn JE, Bell SD, Berger JM. Molecular determinants of origin discrimination by Orc1 initiators in archaea. Nucleic Acids Res 2011;39:3621-31.

[45] Méchali M, Yoshida K, Coulombe P, Pasero P. Genetic and epigenetic determinants of DNA replication origins, position and activation. Curr Opin Genet Dev 2013;23:124-31.

[46] Krause M, Rückert B, Lurz R, Messer W. Complexes at the replication origin of Bacillus subtilis with homologous and heterologous DnaA protein. J Mol Biol 1997;274:365-80.

[47] Alm RA, Ling LS, Moir DT, King BL, Brown ED, Doig PC, et al. Genomic-sequence comparison of two unrelated isolates of the human gastric pathogen Helicobacter pylori. Nature 1999;397:176-80.

[48] Ye F, Brauer T, Niehus E, Drlica K, Josenhans C, Suerbaum S. Flagellar and global gene regulation in Helicobacter pylori modulated by changes in DNA supercoiling. Int $\mathrm{J}$ Med Microbiol 2007;297:65-81.

[49] Ozaki S, Katayama T. Highly organized DnaA-oriC complexes recruit the single-stranded DNA for replication initiation. Nucleic Acids Res 2012;40:1648-65.

[50] Kaur G, Vora MP, Czerwonka CA, Rozgaja TA, Grimwade $\mathrm{JE}$, Leonard AC. Building the bacterial orisome: high-affinity DnaA recognition plays a role in setting the conformation of oriC DNA. Mol Microbiol 2014;91(6):1148-63.

[51] Weigel C, Messer W, Preiss S, Welzeck M, Morigen, Boye E. The sequence requirements for a functional Escherichia coli replication origin are different for the chromosome and a minichromosome. Mol Microbiol 2001;40:498-507.

[52] Briggs GS, Smits WK, Soultanas P. Chromosomal replication initiation machinery of low-G + C-content Firmicutes. J Bacteriol 2012;194:5162-70.

[53] Moriya S, Firshein W, Yoshikawa H, Ogasawara N. Replication of a Bacillus subtilis oriC plasmid in vitro. Mol Microbiol 1994;12:469-78.

[54] Soni RK, Mehra P, Mukhopadhyay G, Dhar SK. Helicobacter pylori DnaB helicase can bypass Escherichia coli DnaC function in vivo. Biochem J 2005;389:541-8.

[55] Sasse-Dwight S, Gralla JD. Footprinting protein-DNA complexes in vivo. Methods Enzymol 1991;208:146-68.

[56] Schneider CA, Rasband WS, Eliceiri KW. NIH Image to ImageJ: 25 years of image analysis. Nat Methods 2012;9:671-5. 